The upgrade of the CMS RPC system during the first LHC long shutdown

This article has been downloaded from IOPscience. Please scroll down to see the full text article.

2013 JINST 8 T02002

(http://iopscience.iop.org/1748-0221/8/02/T02002)

View the table of contents for this issue, or go to the journal homepage for more

Download details:

IP Address: 157.193.98.16

The article was downloaded on 20/06/2013 at 09:04

Please note that terms and conditions apply. 


\title{
The upgrade of the CMS RPC system during the first LHC long shutdown
}

\author{
M. Tytgat, ${ }^{a, 1}$ A. Marinov, ${ }^{a}$ P. Verwilligen,,${ }^{a, 2}$ N. Zaganidis, ${ }^{a}$ A. Aleksandrov, ${ }^{b}$ \\ V. Genchev, ${ }^{b}$ P. laydjiev, ${ }^{b}$ M. Rodozov, ${ }^{b}$ M. Shopova, ${ }^{b}$ G. Sultanov, ${ }^{b}$ Y. Assran, ${ }^{c}$ \\ M. Abbrescia, ${ }^{d}$ C. Calabria, ${ }^{d}$ A. Colaleo, ${ }^{d}$ G. laselli, ${ }^{d}$ F. Loddo,${ }^{d}$ M. Maggi, ${ }^{d}$ \\ G. Pugliese ${ }^{d}$ L. Benussi, ${ }^{e}$ S. Bianco,${ }^{e}$ M. Caponero, ${ }^{e}$ S. Colafranceschi, ${ }^{e, i}$ F. Felli, ${ }^{e}$, \\ D. Piccolo, ${ }^{e}$ G. Saviano, ${ }^{e}$ C. Carrillo, ${ }^{f}$ U. Berzano, ${ }^{g}$ M. Gabusi, ${ }^{g}$ P. Vitulo, ${ }^{g}$ M. Kang,${ }^{h}$
} K.S. Lee, ${ }^{h}$ S.K. Park, ${ }^{h}$ S. Shin, ${ }^{h}$ A. Sharma ${ }^{i}$

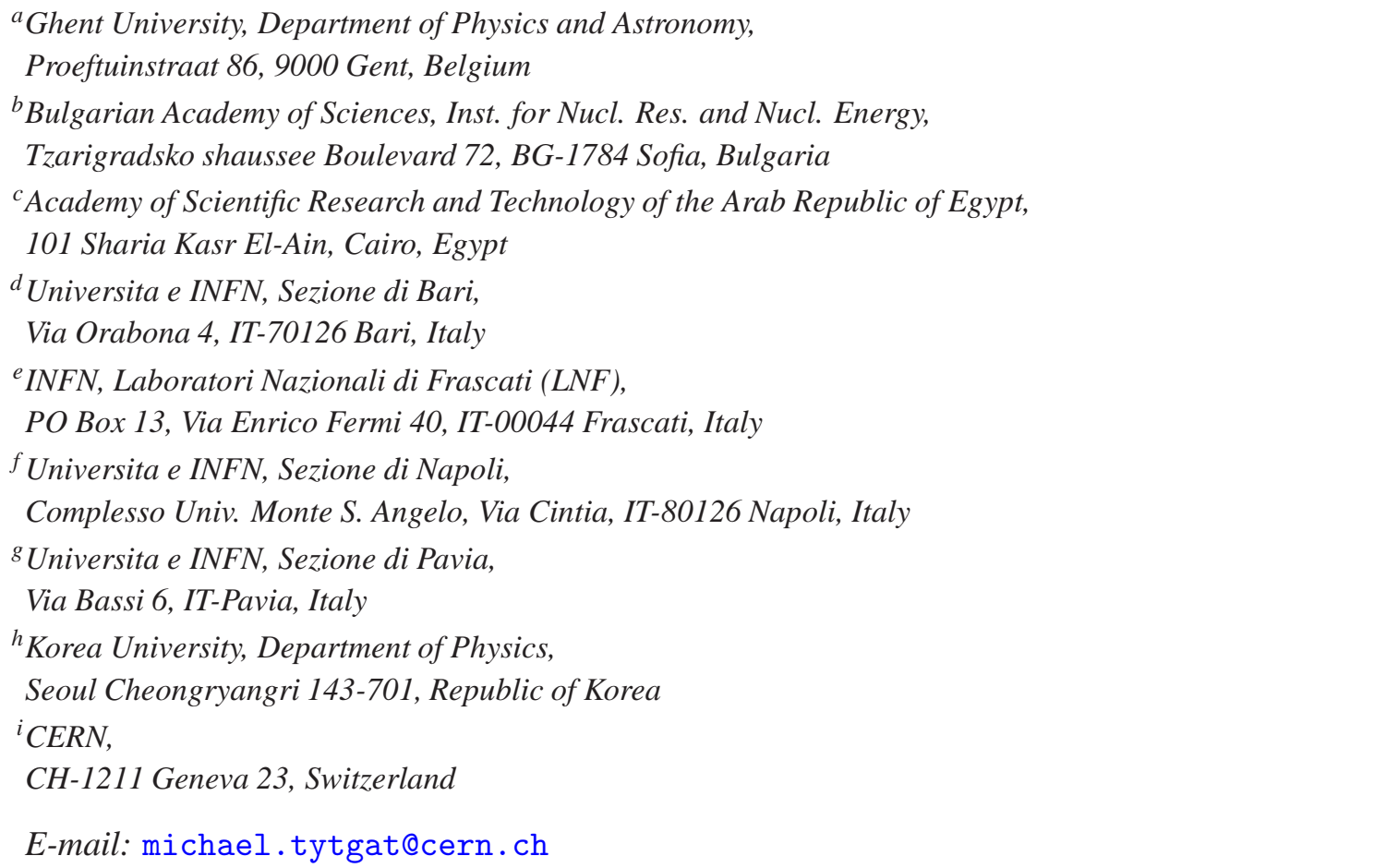

\footnotetext{
${ }^{1}$ Corresponding author.

${ }^{2}$ Now at Universita e INFN, Sezione di Bari.
} 
ABSTRACT: The CMS muon system includes in both the barrel and endcap region Resistive Plate Chambers (RPC). They mainly serve as trigger detectors and also improve the reconstruction of muon parameters. Over the years, the instantaneous luminosity of the Large Hadron Collider gradually increases. During the LHC Phase 1 ( $\sim$ first 10 years of operation) an ultimate luminosity is expected above its design value of $10^{34} \mathrm{~cm}^{-2} \mathrm{~s}^{-1}$ at $14 \mathrm{TeV}$. To prepare the machine and also the experiments for this, two long shutdown periods are scheduled for 2013-2014 and 2018-2019. The CMS Collaboration is planning several detector upgrades during these long shutdowns. In particular, the muon detection system should be able to maintain a low- $p_{T}$ threshold for an efficient Level-1 Muon Trigger at high particle rates. One of the measures to ensure this, is to extend the present RPC system with the addition of a 4th layer in both endcap regions. During the first long shutdown, these two new stations will be equipped in the region $|\eta|<1.6$ with 144 High Pressure Laminate (HPL) double-layer RPCs operating in avalanche mode, with a similar design as the existing CMS endcap chambers. Here, we present the upgrade plans for the CMS RPC system for the fist long shutdown, including trigger simulation studies for the extended system, and details on the new HPL production, the chamber assembly and the quality control procedures.

KEYWORDS: Resistive-plate chambers; Muon spectrometers; Gaseous detectors

ARXIV EPRINT: 1209.1979 


\section{Contents}

1 Introduction 1

2 Motivation for the RPC upgrade 2

3 Design of the new CMS endcap RPCs 2

$4 \quad$ RPC production and quality control $\quad 4$

5 Summary 6

\section{Introduction}

Since the start of the first Large Hadron Collider (LHC) physics run in 2009, the Compact Muon Solenoid (CMS) experiment [2] has been collecting data successfully. During Phase 1 of the LHC which will continue until about 2022, the instantaneous luminosity delivered to the experiments is foreseen to increase gradually up to more than twice its nominal value of $10^{34} \mathrm{cms}^{-2} \mathrm{~s}^{-1}$. At present, two shutdown periods are scheduled to give the machine and the experiments the necessary time to anticipate these luminosity increases: Long Shutdown 1 (LS1) in 2013/2014 should prepare the accelerator to run at its nominal luminosity and Long Shutdown 2 (LS2) in 2018/2019 should take it to 2.2 times this value.

During these long shutdown periods the CMS Collaboration intends to upgrade several subsystems of its detector [3]. In particular, the instrumentation of the muon system as depicted in figure 1, will be extended in both endcaps to ensure efficient muon triggering and reconstruction in that region at high luminosities. In the endcaps, CMS is using Cathode Strip Chambers (CSCs) as muon tracking and trigger detectors, while Resistive Plate Chambers (RPCs) serve as dedicated trigger detectors and improve the muon reconstruction. At present, the 4th endcap disks remain largely uninstrumented: CSCs are only installed in the very forward region $(1.8<|\eta|<2.4)$, and RPCs are missing completely and cover only the first three endcap disks up to $|\eta|=1.6$.

During LS1 these 4th endcap stations will be instrumented further with new CSCs below $|\eta|=1.8$ and new RPCs up to $|\eta|=1.6$. In the following, we describe the CMS plans for this first step in the upgrade of the RPC endcap system.

For the latter subsystem, the very forward region beyond $|\eta|=1.6$ will still remain empty after LS1 and could in principle in a second step be instrumented up to $|\eta|=2.4$ matching the CSC system. However, the present design of the endcap RPCs, made of a double High Pressure Laminate (HPL) gas gap and operating in avalanche mode, is not expected to be suitable for the particle rates amounting to several tens of $\mathrm{kHz} / \mathrm{cm}^{2}$ in the scenario of an LHC luminosity going up to $10^{34-35} \mathrm{~cm}^{-2} \mathrm{~s}^{-1}$. Dedicated R\&D activities to identify suitable technologies to instrument that particular endcap region during LS2 are ongoing, see for instance $[4,5]$. 

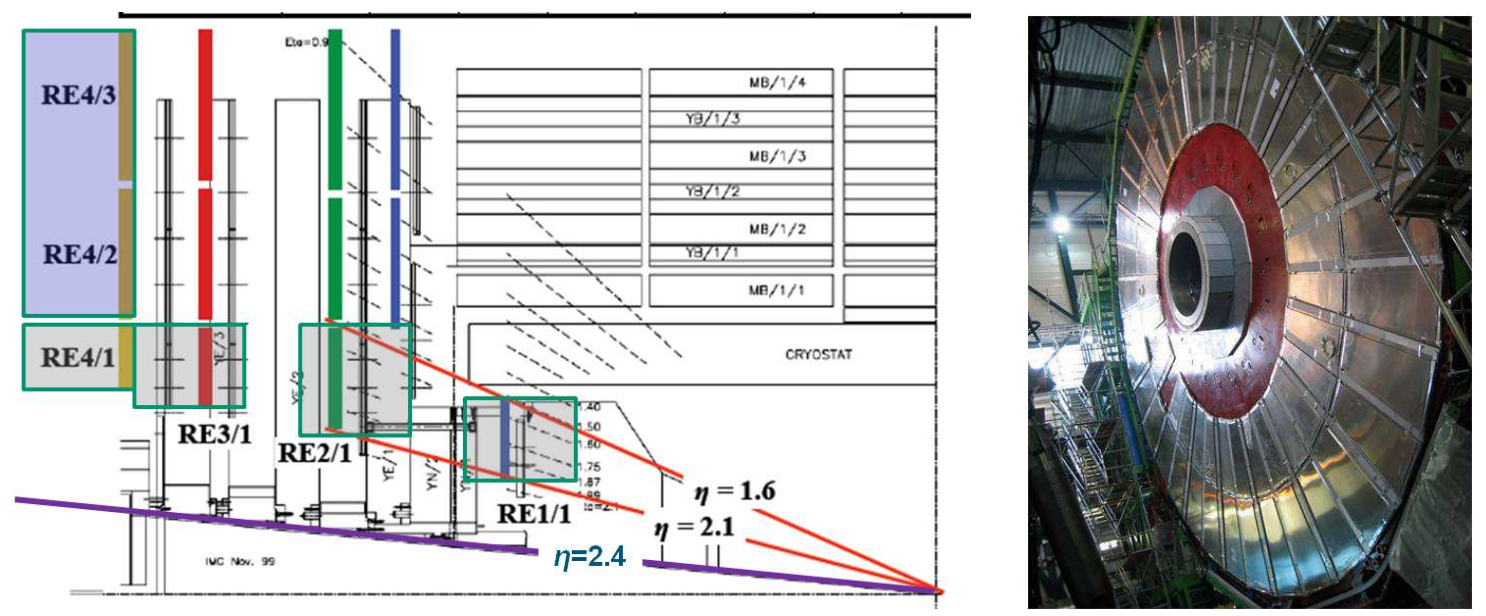

Figure 1. (left) One quarter of the CMS muon system. The colored detectors are the RPCs in the endcap system. RPCs enclosed in boxes have yet to be installed. (right) Photograph of endcap RPC detectors taken during installation of the CMS detector.

\section{Motivation for the RPC upgrade}

Muons with high transverse momenta are one of the key objects in the detection of possible new physics phenomena. By 2015, the LHC luminosity should reach $10^{34} \mathrm{~cm}^{-2} \mathrm{~s}^{-1}$. The in-time pileup will be right at the edge of the CMS design envelope and will present special challenges for the muon system to trigger on high- $p_{T}$ muons. The RPC upgrade is essentially driven by the impact of the instantaneous peak luminosity on the trigger system. In the endcaps, the RPC system provides excellent timing with a somewhat worse momentum resolution compared to the CSC system. To be effective, the muon trigger must achieve good enough resolution to identify high- $p_{T}$ tracks. With the RPC trigger requiring segments in at least three stations, the endcap system does not have the necessary redundancy to control the trigger rate at the increased luminosity while preserving high trigger efficiency. The problem stems from mis-measurements of low- $p_{T}$ muons, that are promoted to high- $p_{T}$ muons and contribute to the trigger rate. With the much higher flux of low-momentum muons at increased luminosity, such poorly measured muons will dominate the trigger rate and make it unacceptably high, leading in turn to unacceptably high muon- $p_{T}$ trigger thresholds.

In figure 2 the RPC Level-1 Trigger performance is simulated for the present system with three stations and the four station situation after the upgrade. The present RPC trigger logic requires hits in at least three layers, which causes the observed drop in efficiency for the endcaps with only three stations. Adding the 4th layer in the endcaps, enabling a 3-out-of-4 trigger logic in those regions, will bring the RPC endcap performance to a similar level as the barrel system.

\section{Design of the new CMS endcap RPCs}

The layout of the CMS RPC endcap stations and chambers is depicted in figure 3. Each station consists of three concentric rings, called $\mathrm{REx} / 1-3$ (station $\mathrm{x}=1,2,3$ ), with chambers mounted in a staggered way. For the instrumentation of the 4th station up to $|\eta|=1.6,144$ new RPCs are re- 


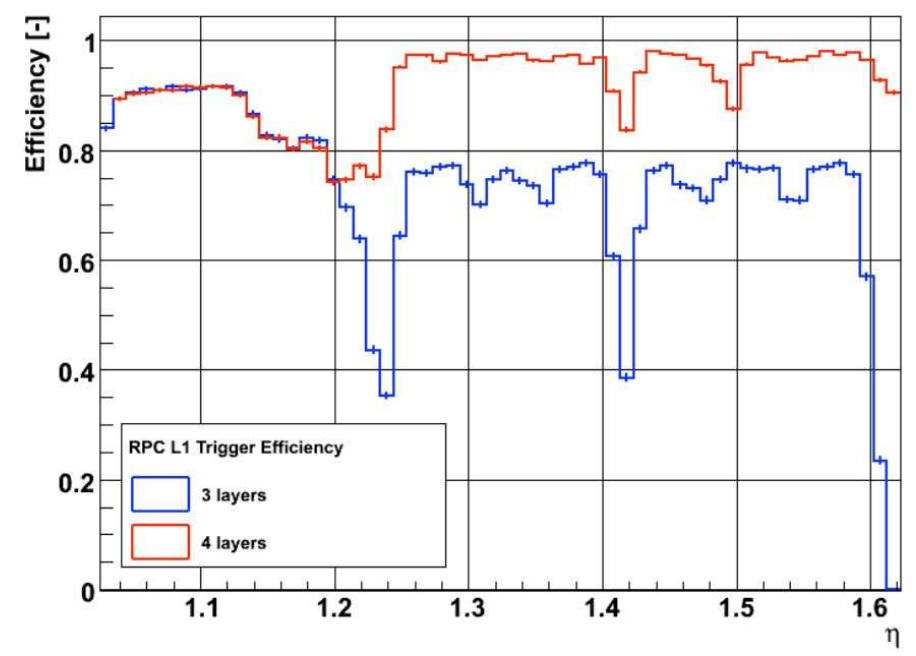

Figure 2. Simulated RPC Level-1 trigger efficiency for the present system with three endcap layers compared to the upgraded situation with four endcap layers.
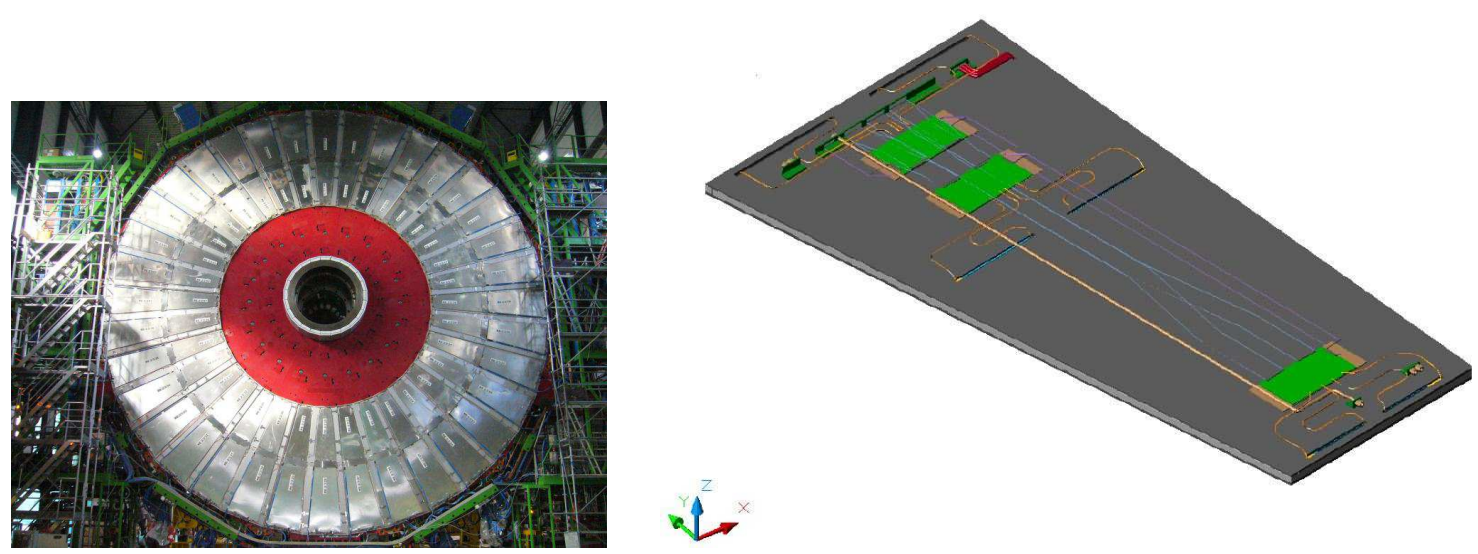

Figure 3. (left) The second RPC endcap station during assembly of the CMS detector. (right) Schematic layout of an endcap chamber.

quired to be mounted in two such concentric rings (RE4/2 and RE4/3) per station, with 36 chambers per ring. As the design of the new chambers is quite similar to the one of the existing RPCs, a total of 200 new chambers will be built with 56 spare chambers for the RE2-4 stations.

As shown in figure 4, the endcap RPCs contain trapezoidal shaped HPL gas gaps, that are organized in a double-layer configuration with a copper strip readout panel placed in between. To simplify the signal cable routing, the design uses one large gas gap at the bottom and two smaller gaps on top. The chamber is embedded in a honeycomb box with the chamber services (readout electronics, gas in and outlets and water cooling circuit) mounted on the outside.

The readout strip panel is divided in $3 \eta$-partitions, with 32 strips each, yielding a total of 96 strips per chamber. Strips are connected through coaxial cables to Adapter Boards linked to 3 Front End Boards (FEBs) per chamber. Every Chamber has 1 Distribution Board for the electronics control. The off-detector electronics consist mainly of Link Boards (LBs) that receive signals 

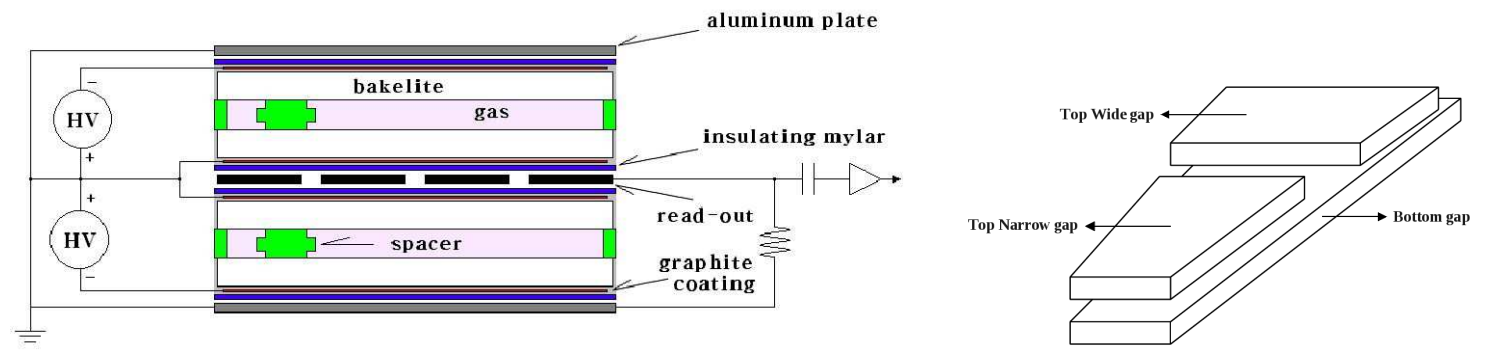

Figure 4. (left) Schematic of the double-layer layout of the endcap chambers. (right) Gas gap configuration of the endcap chambers.

in LVDS (Low-Voltage Differential Signaling) standard from the FEBs. The LBs perform the synchronization with the LHC clock and the transmission to the Trigger Electronics in the control room. Each LB crate contains a Control Board (CB) that drives the crate, provides inter-crate communication, and takes care of the connection to the readout and trigger systems. The new LBs and CBs include minor design improvements related to low voltage regulation and protection circuits, to cure problems observed in the past with the present system.

The working point of the present system depends strongly on the environmental temperature, and the latter should therefore be kept inside $21-23^{\circ} \mathrm{C}$. Due to space limitations for cabling, conventional sensors cannot be used to monitor the temperature. Therefore, each endcap chamber will be equipped with one Fibre Bragg Grating sensor mounted inside a heat conducting housing. These sensors are radiation hard, insensitive to magnetic fields, have a high precision, produce no electrical noise, are easy to install, require a minimal amount of cabling and finally cost about the same as conventional sensors. As the new RE4 RPCs will be mounted inside the CMS detector facing the CSC electronics, there is some danger of additional external heating to the RPC gas gaps. The water cooling circuit on the chambers consists of $\mathrm{Cu}$ pipes soldered onto three copper plates where the FEBs are mounted. An aluminum screen box is covering the cooling circuit and the FEBs. To better stabilize the operating temperature, the layout and size of the copper plates were optimized using FloEFD/pro, ${ }^{1}$ and the final configuration was successfully tested in the laboratory with a detector inside a heated box. In addition, every $10^{\circ}$ sector of the endcap disk will get a connection to the cooling water manifolds in the cavern, i.e. only 2 chambers per connection.

As shown in figure 5, the new RE4 RPCs will be attached on CSC mounting posts on the back of the 3rd endcap yoke of the CMS detector. Before installation, the corresponding RE4/2 and RE4/3 chambers will be pre-mounted together on Super Modules that cover a $10^{\circ}$ sector of an endcap. This will not only reduce the amount of cabling work to be done in the CMS cavern, but will also speed up the installation of the chambers.

\section{RPC production and quality control}

The fabrication of the RPC gas gaps starts with the production of HPL sheets. The raw material is coming from the Puricelli firm (Milano), where about 600 sheets of $1620 \times 3200 \mathrm{~mm}^{2}$ are being

\footnotetext{
${ }^{1}$ FloEFD ${ }^{\mathrm{TM}}$ software package by Mentor Graphics.
} 

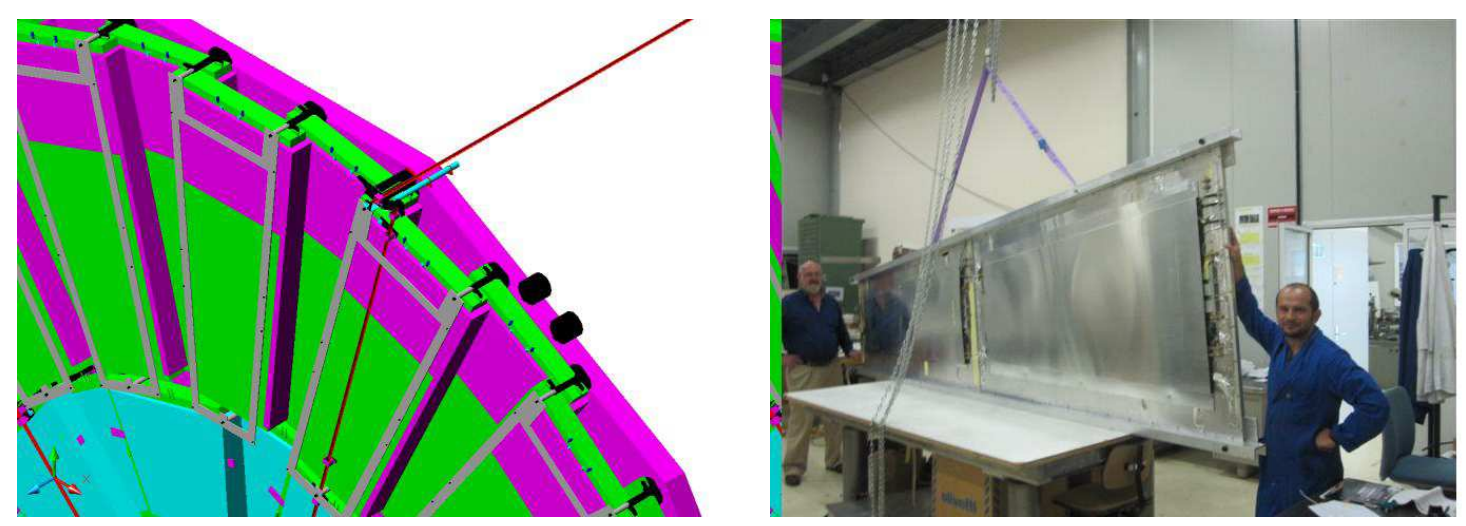

Figure 5. (left) Drawing of the new RPC modules installed on the CSC mounting posts on the backside of the 3rd endcap yoke. (right) Photograph of an RE4 Super Module test installation at CERN.

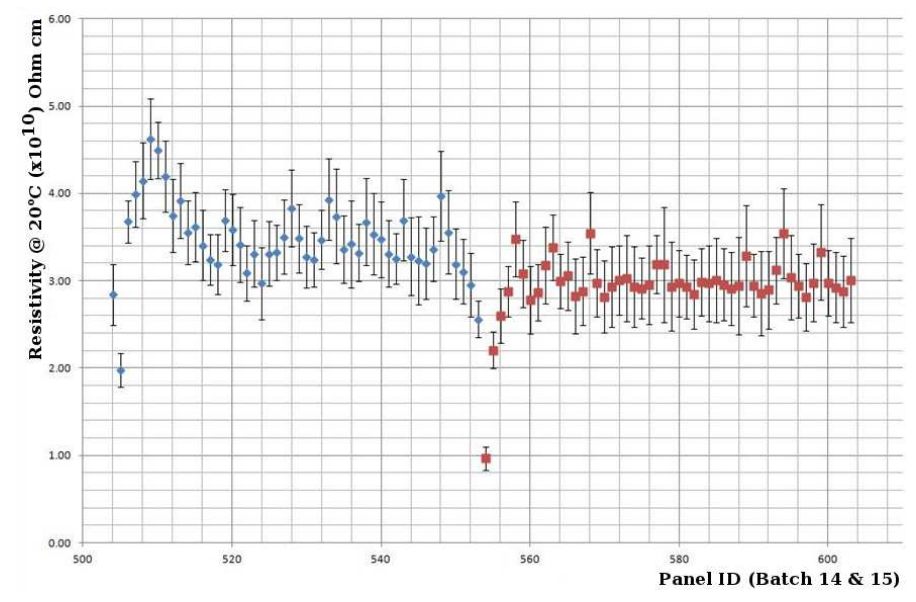

Figure 6. Measured resistivity for recent production batches of HPL sheets. The error bars indicate the spread in resistivity values measured at different points along the surface of the sheet.

produced. The quality control (QC) of the HPL sheets is handled by INFN Pavia. Next, the sheets are cut by Riva (Milano) and finally surface cleaned by General Tecnica (Frosinone).

CMS has put forward detailed specifications for the HPL sheets; main requirements are a high uniformity in resistivity across the surface of the sheets and also between the sheets, with a resistivity value between $1-6 \cdot 10^{10} \Omega \mathrm{cm}$ (measured at $20^{\circ} \mathrm{C}$ ). Figure 6 displays the resistivity values measured for two recent production batches. The data represent the average resistivity value and spread measured at nine different positions across the sheet surface; 50 panels are produced per batch and the obtained resistivity values are clearly uniform and well inside the CMS specifications.

The HPL sheets that pass the QC are sent to the KOrea DEtector Laboratory (KODEL) at Korea University, where about 700 gas gaps will be produced [6]. The HPLs receive a graphite coating, protected with a polyethylene terephthalate (PET) film. Assembled gaps are treated with linseed oil mixed with heptane. Again very detailed technical specifications and QC protocols were prepared by CMS to ensure high quality gaps. Figure 7 shows an example of a HPL gas gap assembled at KODEL. 


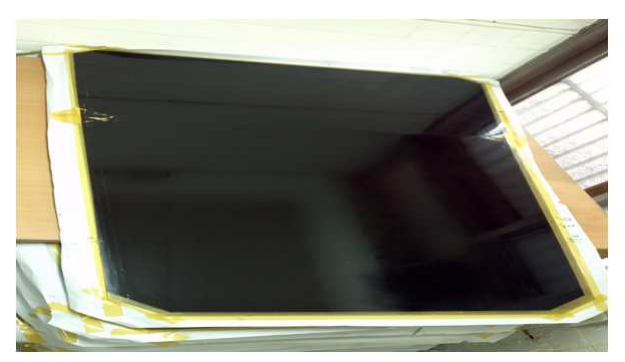

Figure 7. Photograph of a completed HPL gas gap at Kodel.

The chamber mechanics (honeycomb boxes, screen boxes, read-out strip panels, etc.) were produced by two Chinese companies: Beijing Axicomb Technology Co., Ltd and Beijing Gaonengkedi SGT Co., Ltd. In the past, those companies have already produced the same components for the existing system.

The detector electronics are procured in Pakistan. For this project a total of 1350 Adapter Boards, 650 Front End Boards and 250 Distribution Boards will be produced. The new off-detector electronics will be produced by the INFN, while the CMS Warsaw (Poland) group will take on the responsibility to integrate the new electronics in the trigger system.

The final detector assembly is shared by three different institutions: Barc (Mumbai, India), Ghent University (Ghent, Belgium) and CERN. Each of these assembly sites has built up a new RPC lab, including setups for detailed quality control of the HPL gas gaps and completed chambers.

A common quality control protocol for the chamber production has been carefully prepared for each level of the construction. Individual components need to satisfy detailed mechanical specifications. The HPL gas gaps will undergo visual inspections, leak tests and dark current measurements. Assembled chambers will be thoroughly tested (efficiency, cluster size, long term current monitoring, noise) in cosmic test benches at each of the assembly sites. The tests at chamber level will be repeated once the chambers have been transported to CERN and yet again when the chambers have been mounted in the Super Modules. The final checkout will occur after the installation of the Super Modules in the CMS detector.

The full history of each chamber and every component is stored in an Oracle based Construction Database. The database includes all measurements performed before, during and after the detector assembly. This should enable CMS to follow the evolution of each chamber in time, especially in case problems should appear during operation later on.

\section{Summary}

As the instantaneous luminosity of the LHC continues to increase over time during Phase 1, the CMS experiment has to ensure its detector can operate in a stable and efficient manner for increasingly high particle rates. In particular, the muon system should be able to keep its trigger rate and efficiency under control at a low enough muon $p_{T}$-threshold for physics studies. To this end, CMS will among other things extend its RPC system with a 4th layer in both endcaps. The construction of 200 new RPCs of the standard CMS type recently started. Components for the new chambers are 
being produced at various places in the world. The assembly and quality control of the chambers will be handled at three different locations in India, Belgium and CERN. The new chambers are to be installed inside the CMS detector during the first LHC Long Shutdown in 2013-2014.

\section{Acknowledgments}

We acknowledge support from FWO (Belgium); MEYS (Bulgaria); CERN; INFN (Italy); NRF and WCU (Korea); Swiss Funding Agencies (Switzerland).

\section{References}

[1] CMS collaboration, The Muon Project, CMS Technical Design Report, CERN/LHCC 97-32, CMS-TDR-003.

[2] CMS collaboration, The CMS experiment at the CERN LHC, 2008 JINST 3 S08004.

[3] CMS collaboration, Technical Proposal for the Upgrade of the CMS detector through 2020, CERN-LHCC-2011-006, CMS-UG-TP-1, LHCC-P-004.

[4] K.S. Lee, Phenolic Multigap RPCs for high-rate particle triggers in the CMS experiment, in proceedings of RPC2012, XI Workshop on Resistive Plate Chambers and Related Detectors, February, 5-10, 2012 INFN - Laboratori Nazionali di Frascati, Italy.

[5] M. Tytgat et al., Construction and Performance of Large-Area Triple-GEM Prototypes for Future Upgrades of the CMS Forward Muon System, Nucl. Sci. Symp. and Med. Imag. Conf. (NSS/MIC), 2011 IEEE Conf. Record, pp. 1019-1025 [arXiv: 1111.7249].

[6] S. Park, Production of RPC Gaps for the CMS Upgrade, in proceedings of RPC2012, XI Workshop on Resistive Plate Chambers and Related Detectors, February, 5-10, 2012 INFN - Laboratori Nazionali di Frascati, Italy. 